\title{
FORMA Y MOVIMIENTO EN LOS ESTUDIOS LITERARIOS
}

\section{FORM AND CONTENT IN THE LITERARY STUDIES}

\author{
Roy Alfaro Vargas*
}

\begin{abstract}
Así, al examinar el juicio "el movimiento es continuo y discontinuo" desde el punto de vista de su forma, llegamos a la conclusión de que este juicio es contradictorio, aunque por su contenido sea verdadero.
\end{abstract}

Lógica

D. P. Gorski y P. V. Tavants

\section{RESUMEN}

Este artículo analiza las diferentes vías de desarrollo de los estudios literarios, de acuerdo con el patrón lógico del cual partan, por la aplicación de la lógica dialéctica conlleva para los estudios literarios un énfasis en el contenido y su movimiento, mientras que, con la lógica formal, los análisis literarios enfatizan la forma, lo cual deriva en una preponderancia de lo descriptivo.

PALABRAS CLAVE: LITERATURA * LÓGICA DIALÉCTICA * LÓGICA FORMAL * PRINCIPIO DE IDENTIDAD $*$ POIESIS $*$ PRAXIS

\section{ABSTRACT}

This article analyzes the different roads of development of the literary studies, in accordance with the logical pattern of which leave, that is to say, the application of the dialectical logic bears for the literary studies an emphasis in the content and its movement, while, with the formal logic, these they emphasize the form, that derives in a preponderance of the descriptive thing.

KEY WORDS: LITERATURE $*$ DIALECTICAL LOGIC $*$ FORMAL LOGIC $*$ PRINCIPLE OF IDENTITY $*$ POIESIS $*$ PRACTICE

Candidato a la Maestría Académica en Literatura

Latinoamericana, Universidad de Costa Rica.

royalfarov@gmail.com 


\section{INTRODUCCIÓN}

Nuestra reflexión seguirá los derroteros de los límites establecidos en la aplicación de una lógica formal o de una dialéctica al análisis literario, a la vez que se señalarán las divergencias ontológicas implícitas en cada uno de estos enfoques lógicos. Partiremos del principio de identidad lógico-aristotélico, para desarrollar, a partir de tal principio, las ontologías presentes en estas lógicas $y$, en consecuencia, las diferencias metodológicas existentes principalmente alrededor de las categorías de tiempo y espacioํ․ Para lograr esto será preciso:

$\diamond$ Definir el principio de identidad de la lógica formal.

$\diamond$ Establecer las relaciones dadas entre tal principio y las ontologías subyacentes a cada una de estas lógicas.

$\diamond$ Señalar las diferencias metódico-metodológicas ${ }^{2}$ derivadas de la aplicación de estas lógicas, en relación con lo literario.

$\diamond$ Determinar la relación entre cada una de estas lógicas y las categorías de tiempo y espacio, con respecto al estudio de la literatura.

$\diamond$ Definir los espacios de poiesis y de praxis, como elementos característicos de cada de una de esas lógicas, en la literatura.

Es importante recordar que la lógica formal tiene como objeto el pensamiento humano (Gorski, 1970: 14). La lógica formal establece los procesos necesarios "para alcanzar la verdad por medio de un conocimiento inferido"

$1 \quad$ Este escrito va en la misma línea de mis artículos publicados en la Revista de Ciencias Sociales, UCR (Alfaro, 2005a y 2005b). Con estos artículos se pretende montar el aparato teórico-epistemológico de este nuevo enfoque metodológico, el cual intenta sobrepasar la antigua propuesta luckasiana (Lukács, 1968), por lo tanto este artículo no es de carácter empírico.

La noción de método refiere al conjunto de principios ontológico-epistemológicos que guían la investigación y el término metodología agrupa las herramientas, propiamente dichas, utilizadas para el análisis.
(Gorski, 1970: 15). Esta lógica permite aprehender los rasgos estructurales del pensamiento, que caracterizan el razonamiento correcto $y$ que, por consiguiente, lo diferencian del incorrecto. Para esto, la lógica formal aplica tres principios o leyes: el principio de identidad, el de contradicción y el de tercero excluido ${ }^{3}$.

La lógica dialéctica, alejándose del esquema ontológico de la lógica formal, concibe el Ser (real, concreto) como la suma de la identidad más negatividad (Kojève, 1972: 42). La dialéctica es la lógica de la negatividad, o sea, de lo posible, ya que no se dedica a describir lo dado (como las metodologías ancladas en el principio de identidad) sino que, a partir de lo dado, propone posibles vías de desarrollo insertas en lo concreto. La dialéctica no es una lógica que se centre en los procesos para producir o evaluar la corrección de un razonamiento, sino la superación (Aufhebung) del establishment. En este marco, la dialéctica es una lógica de la relación, del conflicto y una lógica de la vida (Garaudy, 1974: 49, 51, 53 y 55). La dialéctica es una vacuna contra las posturas teleológicas de la historia ${ }^{4}$ (Seoane, 2000:212).

\section{EL PRINCIPIO DE IDENTIDAD}

El principio de identidad es $\mathrm{A}=\mathrm{A}$, o sea, que un fenómeno es conceptualmente siempre idéntico a sí mismo. Por ejemplo: el texto Los Peor (1998) de Fernando Contreras es, si y solo si, se manifiesta en sí. El concepto es idéntico a sí mismo siempre y cuando su extensión sea

Confróntese: (Gorski, 1970: 307-316). Algunos lógicos incluyen un cuarto principio, el de razón suficiente (Gorski, 1970: 315). El principio de identidad conforma una noción ontológica en la cual el Ser es definido como identidad $(\mathrm{A}=\mathrm{A})$, o sea, como lo dado (Kojève, 1972: 46). Como veremos más adelante la dialéctica es la superación de este esquema ontológico de cuño lógico formal.

$4 \quad$ El lector puede consultar los textos de: Copi (1977), Kopnin (1966), Adorno (1975), Lefebvre (1976), Gortari (1984) y Hegel (1973 y 1976), para profundizar en los aspectos relevantes de la definición de cada una de estas lógicas y en su correlación. 
la misma, es decir, mientras no se afecte su intensión (los rasgos esenciales que permiten agrupar X fenómenos dentro de tal concepto) ${ }^{5}$. Dicho brevemente, el principio de identidad implica que "los pensamientos son idénticos entre sí, si poseen la misma extensión; todo pensamiento enunciado es idéntico a sí mismo si su extensión permanece invariable" (Gorski, 1970: 309) ${ }^{6}$. Este principio implica que el objeto al cual refiere el concepto o no cambia, o simplemente nos abstraemos de su proceso de cambio (Gorski, 1970: 307). Este principio funda una lógica estática, a la cual el movimiento le es ajeno.

El principio de identidad es el punto de inserción de la crítica de la lógica dialéctica, la cual es una lógica del movimiento. Pero es la relación de cada una de las lógicas señaladas la que establece "por debajo" una ontología totalmente opuesta a la de la otra lógica.

\section{ONTOLOGÍA, LÓGICA FORMAL Y LÓGICA DIALÉCTICA}

La lógica formal, mediante el principio de identidad, se funda en y fundamenta una ontología rígida y positiva, ya que dos objetos o conceptos solamente pueden ser idénticos si se apela a su valor formal. Exempli gratia: Los Peor solo puede ser él mismo si se cuantifican una serie de elementos formales, como sus características clasificatorias (ser manifestación de la promoción del 80 o tener determinados elementos posmodernos) o como sus elementos formales (la técnica esperpéntica). De esta manera, los juicios, al respecto de Los Peor,

De acuerdo con Eli de Gortari, "los conceptos científicos poseen siempre un contenido concreto. (...) El aspecto cualitativo de ese contenido recibe el nombre de intensión del concepto. Y el aspecto cuantitativo de ese mismo concepto constituye la extensión del concepto. La intensión significa el grupo de cualidades de los procesos que el concepto contiene en su determinación. La extensión, en cambio, indica la cantidad de procesos a los cuales refiere la determinación del concepto" (Gortari, 1984: 12).

En cursiva en el original. siempre refieren a un concepto cuya extensión está dada por el texto mencionado, es decir, Los Peor en sí.

El en sí de la lógica formal es lo dado, lo establecido, el statu quo; evidentemente lo positivo: lo empírico, lo comprobable, lo cuantificable, lo perceptible, lo presentificable como dato $y$, en último caso, lo concreto (el eîdos). 0 sea, la lógica formal permite reflexionar sobre lo concreto.

La lógica dialéctica, en cambio, reflexiona a partir de lo concreto (Adorno, 1975: 41), es decir, esta lógica establece una ontología en donde el soporte no es el ser (lo concreto, lo dado), sino la unidad dialéctica entre Ser y deber ser. En otras palabras, es una ontología que establece una tensión entre lo real y lo posible, en tanto movimiento del contenido dado como praxis. La dialéctica no es descriptiva, es crítica, debido a que su objeto se mueve y su énfasis es lo continental, $y$ no lo formal. El en sí y el para sí se articulan en una indisoluble relación unitaria por oposición, en donde, durante el devenir, la forma y el contenido están en constante superación (Aufhebung) del uno con respecto al otro.

Del esquema $A=A$ de la lógica formal, se pasa a uno donde A deviene no-A, por lo tanto su opuesto. Si partiésemos de lo literario llegaríamos a lo no-literario, de Los Peor a lo no-Los Peor. De ahí que dentro del esquema dialéctico no se discuten las características del texto literario (Fokkema, 1992: 125).

\section{DIFERENCIAS METÓDICO-METODOLÓGICAS DERIVADAS DE LA APLICACIÓN DE LA LÓGICA FORMAL O DE LA LÓGICA DIALÉCTICA}

La asunción de una u otra lógica implicará la aplicación de principios epistemológicos $y$ de estrategias metodológicas, que se oponen mutuamente a sus respectivos principios, por cuanto la relación que se establece con lo concreto, a partir de cada una de estas lógicas, es incompatible con la de la otra lógica, ya que una reflexiona sobre lo concreto (en una ontología del en sí) y la otra, lo hace a partir de lo concreto (en una ontología de la unidad del en sí-para sí). 
El estructuralismo es una manifestación metodológica de la lógica formal. Para el estructuralismo, la literatura se concibe como un sistema funcional, en donde el término constante es el concepto de obra (Fokkema, 1992: 77). La obra es una estructura en la cual se establece un complejo de relaciones de interdependencia, entre las partes y el todo (Broekman, 1974: 12). Por ende, la obra se define como un sistema cerrado (en términos de von Bertalanffy) o como inmanencia (en términos estructuralistas).

$\mathrm{La}$ inmanencia sigue un esquema lógico formal, en donde la literatura, en general, $y$ la obra literaria, en particular, se definen a partir de sí mismas, en cuanto una serie de rasgos intencionales que, de hecho, determinan la extensión del concepto estructural de literatura. Por tanto, la suma de factores actanciales, de isotopías y funciones (por ejemplo) determina la extensión del concepto de obra.

La aplicación del principio de identidad, en el estructuralismo, se da dentro de las exigencias de la objetividad del paradigma positivista (de corte comtiano) y dentro de una gama de separaciones ontológicas (diacronía/ sincronía, significante/significado) que recuerdan la separación cartesiana de res extensasres cogitans.

Con los postestructuralismos, posteriormente, hay un desplazamiento de la inmanencia de la obra, al contexto (de escritura o de lectura); con lo que la obra deviene texto. Por ejemplo, dentro de los estudios poscoloniales, autores como Gayatri Spivak y Homi Bhabha, con una perspectiva derridiana, intentan deconstruir el eurocentrismo presente en los procesos identitarios de las excolonias (NagyZekmi, 2003: 12).

La perspectiva derridiana conlleva que un texto (A) puede ser interpretado de diversas maneras, tales que todas las interpretaciones son válidas. Así, las interpretaciones X, Y y Z, que derivan de $\mathrm{A}$, dependen, en lo respectivo a su validez, del posicionamiento del interpretante. Intentando poner el énfasis en el proceso de interpretación, se cree que el efecto substancialista del enfoque estructuralista, dado por el principio de identidad lógico-formal, se supera. Dentro de una relación de igualdad todos los términos se igualan ${ }^{7}$ (se homogenizan, para usar un término muy posmoderno). $\mathrm{Si}$ del texto A se derivan las interpretaciones $\mathrm{X}$, Y y $\mathrm{Z}$, entonces cada una de estas interpretaciones remite a A; luego, estas interpretaciones son elementos intensionales del texto A y, por ende, a pesar del factor fenomenológico ${ }^{8}$ dado en el acto hermenéutico, se cae en lo positivo, ya que el elemento extensional es, nuevamente, el texto en sí: $\mathrm{A}=\mathrm{X}, \mathrm{Y}, \mathrm{Z}$ que son la misma manifestación del segundo término de la igualdad (es decir A; X, Y, Z=A, el asunto es conmutativo). Lo concreto eidético sigue siendo el punto de referencia tanto para estructuralistas, como para postestructuralistas.

Es importante recalcar que, con respecto a estas metodologías estructuralistas $y$ postestructuralistas, el paradigma epistemológico (o sea, los principios del método) quedan separados (pero funcionando) de lo metodológico.

Tanto estructuralistas, como postestructuralistas, comparten y conservan del positivismo la idea de la especificidad del objeto ( $y$ del discurso) de cada ciencia. Esto por cuanto, dentro del esquema positivista, la realidad (la Totalidad) se descompone en varias partes, de las cuales deriva el objeto de estudio de cada diferente ciencia positiva; por ejemplo, a la psicología le corresponde el comportamiento humano a partir de lo individual, a la sociología los procesos de integración social, a la crítica literaria el manejo formal del lenguaje y así sucesivamente. Cada ciencia tiene un objeto que es el marco extensional e intensional que la define. Como veremos más adelante, esto no es válido dentro del marco de la lógica dialéctica.

Dentro de estas nociones lógico-formales $y$ en relación con el concepto de especificidad, el análisis positivo (positivista o fenomenológico) se acerca al texto literario $y$, en función de su problema-hipótesis, va señalando los elementos pertinentes para la prueba de la hipótesis, para luego interpretar lo hallado en el texto en sí con base en el marco teórico previamente

Valga la redundancia, para acentuar el efecto al que referimos.

$8 \quad$ La fenomenología es positivismo (Villalobos, 1999: 408). 
apuntado. Villegas, por ejemplo, desarrolla una metodología de análisis teatral en la cual se estudia la especificidad de la obra dramática, en donde tal especificidad consiste en el carácter apelativo del lenguaje, en tanto su virtud es lo dramático (1971: 21). Esta especificidad es aprehendida mediante el examen de fases $y$ subfases que permiten describir el encadenamiento de las situaciones dramáticas que crean el efecto dramático. Sin embargo, luego, hay una exigencia interpretativa que radica en la necesidad de aclarar la ideología subyacente a la obra, la cual sirve para alterar o influir sobre el mundo circundante $y$, además, es preciso explicar el texto como producto autónomo (Villegas, 1971: 26-27).

El marco teórico-conceptual es el encargado de brindar las herramientas para realizar este acto explicativo, es decir, lo abstracto permite la comprensión (Verstehen) de lo concreto. Por tanto, primero dictamino los datos pertinentes para mi hipótesis en el texto en sí y después los explico y comprendo mediante un marco teórico-conceptual, el cual nos permite acceder a la especificidad ideológico-literaria del texto en sí. De lo abstracto vamos a lo concreto, recordemos esto.

La lógica dialéctica, al aplicarse al estudio de la literatura, sigue caminos muy diferentes a los establecidos en las metodologías positivas, cuyo afán descriptivo-explicativo y/o hermenéutico las llevan a circunscribirse alrededor del principio de identidad de la lógica formal.

La dialéctica, siguiendo a Lefebvre, "No es tanto un método de análisis como un método de construcción sintético y sistemático del contenido"9 (1974: 55). La lógica dialéctica no describe elementos formales y luego los interpreta, ella parte de lo concreto ${ }^{10}$ (la forma), a través de un sujeto (el hombre social) que posee ya determinados medios (conocimientos y experiencias), en tanto principio activo que actúa sobre el objeto (Kopnin, 1966: 394), en tanto

"Es necesario partir del contenido. El contenido tiene la primacía; es el ser real el que determina al pensamiento dialéctico" (Lefebvre, 1974: 91). "método de búsqueda de nuevas tesis científicas (conceptos, teorías)" (Kopnin, 1966: 459). Este método no se centra en lo dado, sino en la superación (Aufhebung) de ello, en cuanto praxis, o sea, como lógica del movimiento y construcción del contenido. Para esto, "El investigador debe empezar el estudio del objeto (literario) por el final, por la forma más madura; en ella aparecen más desarrollados sus aspectos esenciales y no están ocultos" (Kopnin, 1966: 186) ${ }^{11}$, lo cual rompe con la linealidad de la causalidad positivista y con la relación aplicativa de las categorías de lo abstracto y lo concreto dentro de la lógica formal. Sin embargo, la elaboración de una forma negativa (en tanto superación -Aufhebung - de lo positivo) conlleva para las categorías de lo abstracto y lo concreto un cambio con respecto a su empleo, dentro del esquema lógico-aristotélico; y también una reestructuración de las relaciones lineales de la causalidad positiva.

Dicho esto de manera más práctica, el investigador se posesiona sobre un texto literario $\mathrm{A}$, que es lo concreto, portando consigo una serie de conocimientos y experiencias (lo abstracto). Pero, a diferencia de lo valorado dentro del esquema lógico formal, aquí no se versa sobre lo concreto. Por ende, los rasgos intensionales no refieren al texto en sí, sino al texto para sí, o sea, la nueva forma que contiene un nuevo contenido (el cual estaba en el texto en sí como lo negado) es toda otra cosa que lo concreto inicial. Se parte de A y se establece un número determinado de rasgos intensionales (X, Y y Z) que remiten a no-A, el opuesto dialéctico de $\mathrm{A}^{12}$. $\mathrm{Y}$ después de todo este movimiento de A hacia no-A, las relaciones de causalidad, lineales y unidireccionales de la positividad, se bidireccionalizan. El objeto A, desde donde se partió para la construcción de no-A, es la causa, por tanto, de su negación (no-A), pero no-A, a la vez, permite explicar a A, es decir, los motivos para que A se manifieste así y no de

\section{Paréntesis mío.}

12

Para un pensamiento positivo a ultranza, esto es un sacrilegio, ya que lo empíricamente dado es anulado en el movimiento de la superación (Aufhebung) dialéctica: todo es pura negatividad. 
otra manera: no-A es la causa de la necesidad de A. De lo abstracto (el conocimiento previo) vamos a lo concreto (el texto en sí) y, luego, nuevamente, a lo abstracto (el texto para sí): del texto en sí solo persiste su negación. En la dialéctica de lo abstracto y lo concreto, es imperativo, con respecto al quehacer del investigador, el teorizar, ya que, con el paso de lo concreto a lo abstracto, lo abstracto inicial se ha modificado por la negación que le imprime lo concreto $y$, por ende, esta modificación implica retomar el conocimiento del inicio e introducirle los cambios operados en la primera negación de lo concreto. 0 sea, el segundo movimiento de lo concreto a lo abstracto es la negación de la negación que, primeramente, lo concreto había hecho de lo abstracto.

Y de la metodología, solo se puede decir que, en la dialéctica, es un determinado enfoque válido para el estudio del fenómeno ${ }^{13}$ (Kopnin, 1966: 102), ya que la idea de una metodología está fundida dentro del esquema categorial de esta lógica, cuya base es lo abstracto y lo concreto (Kopnin, 1966: 84). En la dialéctica, dicho muy didácticamente, si necesito aprehender una escena en un momento específico, usaré una cámara fotográfica; pero si preciso abarcar no un

Al respecto de la metodología y del método, el lector puede consultar los artículos de mí autoría: (Alfaro, 2005a y 2005b). En ellos, se establecen algunas pautas del enfoque sociológico literario, que tiene como fundamento la dialéctica. El objeto de esta sociología dialéctica de la literatura se define como "el análisis dialéctico de la relación individuo-sociedad, a través de la asunción de la literatura como mediación de esa relación, en tanto actividad socializante, en el marco de una integración social dada en el conflicto de la lucha de clases del capitalismo globalizante. Esto en el marco del concepto de negatividad hegelianomarxiano..." (Alfaro, 2005a: 76). Dicho de otra manera, la sociología dialéctica de la literatura se inserta en un vacío teórico-práctico mediante la aplicación de la negatividad al análisis literario, en donde lo narratológico — que es una expresión positiva-queda a merced de la superación dialéctica (Aufhebung). Además, dentro de una línea dialéctica remitimos al lector a considerar los siguientes textos: (Mora, 1991) y (Roth, 2006), en donde se hallarán dos propuestas materialistas ligadas a la hermenéutica (en el primer caso) y a la semiótica (en el segundo caso). punto en la onda del movimiento, sino la onda en su cambio, emplearé una cámara de vídeo.

\section{TIEMPO Y ESPACIO EN RELACIÓN CON LA LÓGICA FORMAL Y CON LA LÓGICA DIALÉCTICA, EN EL QUEHACER DE LOS ESTUDIOS LITERARIOS}

Las categorías de tiempo y espacio son los conceptos básicos ${ }^{14}$ de todo proceso cognitivo-epistemológico, debido a que todo objeto-sujeto se ubica en determinadas coordenadas representables como tiempo y espacio. Un texto literario es escrito en Costa Rica el 27 de diciembre del 2006 y puede ser objeto de estudio para alguien en París en febrero del 2008. Siempre estamos atravesados por el tiempoespacio. Pero, ¿cuál tiempo y cuál espacio? Y en ¿cuál lógica se ubican ese tiempo y ese espacio? Son las mismas categorías de tiempo y espacio válidas para la lógica dialéctica y para la formal.

Si la lógica formal tiende a establecer entidades fijas, mediante el principio de identidad, y la lógica dialéctica anula o supera tal principio, a favor del movimiento del contenido, entonces la relaciones tiempo-espacio tienen que asumirse de diferente manera según se ponga énfasis en la forma o en el contenido. Luego, tiempo y espacio tienen diferentes valores, debido a que, exempli gratia, las entidades fijas de la lógica formal solo pueden existir dentro de un tiempo substancial o absoluto. Veamos esto con más detalle.

El texto en sí es, si y solo si, sigue siendo sí-mismo, para cualquier posición espacial. O sea, Los Peor de Fernando Contreras es el mismo texto en Costa Rica en el 2006, que en París en 2002 o que en la misma Costa Rica en 1998. Pero el texto en sí solo puede ser él-mismo como forma, ya que, el contenido cambia en función del desarrollo histórico de la lucha de clases y de los procesos de socialización en los que se inserte dentro de cada sociedad en particular. Por tanto, solamente a nivel fenoménico (como forma) el texto puede subsistir

Ya Kant ha explicado de sobra la importancia, en la fundamentación del aparato categorial, de las categorías de tiempo y espacio; cfr.: (Kant, 1973: 567) 
en sí. Este aspecto fenoménico salva de caer en contradicciones, ya que, si el texto A es siempre A, entonces no-A es imposible.

La aproximación lógico-formal al estudio de la literatura se enmarca dentro de los derroteros de la física mecánica ${ }^{15}$, ya que, dentro de esta física el tiempo es absoluto. Esta es una concepción ligada a Aristóteles y a Newton, para quienes "El tiempo estaba totalmente separado y era independiente del espacio" (Hawking, 1988: 37). La absolutización del tiempo reduce el objeto literario a sus elementos formales, a una entidad espacial, sobre la cual el tiempo no actúa materialmente ${ }^{16}$ hablando.

La lógica dialéctica apunta en otra dirección. El texto aquí está inmerso en el tiempo, en tanto el contenido es el punto de referencia, en cuanto él es construido por la acción del sujeto. El paso de A a no-A hace que la existencia espacial del texto (que es un objeto con propiedades físicas - peso, medida - y tiene palabras que caen en el espectro sónico o visual) entre en conjunción con el tiempo, es decir, el texto es absorbido por la dinámica del principio de entropía (segunda ley de la termodinámica de Heisenberg $)^{17}$, que se manifiesta como una "flecha del tiempo", o sea, el tiempo va siempre en una progresión positiva. Por ejemplo, si tomo un lápiz en mis manos y lo parto en dos, entonces el sistema, llamado lápiz, se irá degradando cada vez más hasta que se descomponga totalmente. Nunca el lápiz tenderá a reconstituirse: no hay un efecto negentrópico que permita devolver el tiempo, para reconstruir el sistema denominado lápiz. Y, aunque formalmente podamos hablar aún del lápiz, ya este no existe, su contenido ha sobrepasado su formalización lingüística.

Este principio entrópico, en términos dialécticos, indica que de A pasamos a no-A, ya

Bitsakis ya había reflexionado sobre la relación de la física y la dialéctica, en Física contemporánea y materialismo dialéctico (1975).

16 Material en sentido filosófico, es decir, como rasgo dialéctico (cfr.: Kopnin, 1966: 97). que tiempo y espacio se vuelven uno solo ${ }^{18}, y$ cualquier alteración en el tiempo se manifiesta en el espacio y viceversa: se opera una topologización del tiempo, en donde el tiempo se relativiza $y$, por ende, el valor textual de un producto literario depende del lugar que se tenga en el espacio. Sin embargo, esto no es una forma de determinismo fisicista, por cuanto "El espacio y el tiempo no sólo afectan, sino que también son afectados por todo aquello que sucede en el universo" (Hawking, 1988: 56). Por lo tanto, la dinámica social afecta la relación tiempoespacio, lo cual es patente en la lógica formal misma, que crea una escisión entre tiempo y espacio.

El contenido, dado dentro de este fenómeno topológico, permite que lo literario, al ser objeto de estudio, devenga lo no-literario. En otras palabras, se puede iniciar la construcción del contenido partiendo de una novela como Los Peor, la cual puede devenir en una nueva forma que sea no-literaria, es decir, psicológica, sociológica, física, matemática o un conglomerado de todas ellas ${ }^{19}$. El objeto literario concreto se abstrae, para convertirse en una nueva forma, que luego será también abstraída y asî sucesivamente.

\section{ESTÉTICA Y POLÍTICA: DE LA POIESIS A LA PRAXIS}

La lógica formal se levanta sobre una ontología de lo dado, en donde la forma se erige como el sustento de su inmovilidad y el espacio de una descripción que se cierra en un círculo hermenéutico. Mientras tanto la lógica dialéctica se establece como la unidad ontológica

"Debemos aceptar que el tiempo no está completamente separado e independiente del espacio, sino que por el contrario se combina con él para formar un objeto llamado espacio-tiempo" (Hawking, 1988: 43-44).

Esto viene aunado por el concepto de Totalidad (Lukács, 1975) que rechaza la noción de especificidad señalada anteriormente (véase arriba), ya que, para la dialéctica, el objeto, al estar en movimiento, no permite anclársele en el ámbito de una ciencia particular. 
entre ser y deber ser, en tanto constructora del contenido y del movimiento, en cuanto Literaturwissenschaft (ciencia literaria).

La lógica formal establece la poiesis como espacio de legitimación y de verosimilitud, ya que permite el establecimiento de estéticas que conllevan un afán clasificatorio. Por ejemplo, hay una estética naturalista ${ }^{20}$ en donde se señalan algunos elementos formales que deben cumplir los textos, para ser catalogados dentro de las manifestaciones de tal estética. La poiesis es la producción de sentido (Sinn, sens) a partir de una estética de referencia. Es un proceso formal en donde el valor estético de un texto, como por ejemplo Los Peor, depende de las características de la promoción de la década de los 80 y que implica características como: un desencanto posmoderno, el rescate de los particularismos (género, minorías sexuales), la deformación carnavalesca, la metamorfosis y otras características (Quesada, 2000: 2833 y 36). Lo literario se define mediante lo (meta) literario, es decir, el discurso historiográfico de la crítica literaria positiva. La identidad y la especificidad del discurso positivo se re-produce.

La lógica dialéctica se define como praxis. El análisis literario aquí ya no tiende a mantener la especificidad de un objeto, sino que busca insertar el movimiento del contenido dentro de la unidad de la teoría y la práctica, para, dentro del marco de la Totalidad, llegar a la superación (Aufhebung) de lo dado, de lo establecido, del statu quo. La dialéctica no se empeña en comprobar los datos empíricos, más bien los sobrepasa, mediante la asunción de la posibilidad que duerme en la pretendida realidad estática del Ser. No hay un esfuerzo por historiografizar, sino por hacer Historia (Geschichte).

\section{CONCLUSIONES}

La lógica formal y la dialéctica son los lentes con los que aprehendemos la realidad. Y a cada lente, una realidad. De un lado, la realidad estática y formalizada de la lógica formal.
Del otro lado, la realidad móvil, dinámica y dialectizada entre el Ser y el Deber Ser de la lógica dialéctica.

Como en la física, se puede estudiar la luz como onda o como partícula. La física mecánica se centra en la partícula y la física relativa, en la onda, en el movimiento. Por su parte, las metodologías positivas se amarran a la discontinuidad (la partícula, un punto de una recta, lo concreto). En cambio, el método dialéctico aprehende y construye la continuidad a partir de la discontinuidad.

Lógicas para todos los gustos y perspectivas políticas, en donde la dialéctica, al unificar lo continuo y lo discontinuo, se manifiesta como lo general con respecto a lo particular (lo discontinuo) de la lógica formal.

Es deber de cada uno reflexionar sobre las implicaciones políticas de cada una de estas lógicas.

\section{BIBLIOGRAFÍA}

Adorno, Theodor W. Dialéctica negativa. Traducción: José María Ripalda. Madrid: Taurus Ediciones, 1975: 416.

Alfaro Vargas, Roy. "Relación literaturasociedad. Una aproximación teórica”. Revista de Ciencias Sociales 108 (II). Universidad de Costa Rica. 2005a: 71-78.

"Sociología dialéctica de la literatura: cuestión de método". Revista de Ciencias Sociales 109-110(III-IV). Universidad de Costa Rica. 2005b.

Barros, Cristina y Souto, Arturo. Siglo XIX: romanticismo, realismo y naturalismo. $2^{\text {a }}$ edición. México: Editorial Trillas, 1982: 108 .

Bertalanffy, Ludwig von. Teoría general de los sistemas. Traducción: Juan Almeda. Octava reimpresión ( $1^{\text {a }}$ edición). México: Fondo de Cultura Económica, 1991: 312.

Bitsakis, Eftichios. Física contemporánea y materialismo dialéctico. Traducción: José 
Fernández Valencia. México: Ediciones de Cultura Popular, 1975: 268.

Broekman, Jan M. El estructuralismo. Traducción: Claudio Gancho. Barcelona: Editorial Herder. 1974: 204.

Contreras, Fernando. Los peor. $1^{\mathrm{a}}$ edición $\left(2^{\mathrm{a}}\right.$ reimpresión). San José, Costa Rica: FARBEN. 1998: 248.

Copi, Irving. Introducción a la lógica. $18^{\mathrm{a}}$ edición. Traducción: Néstor Alberto Miguez. Buenos Aires: Editorial Universitaria, 1977: 616.

Davies, Paul. "La flecha del tiempo". Investigación y ciencia 314, noviembre. 2002a: 8-13.

"La máquina del tiempo". Investigación y ciencia 314, noviembre. 2002b: 16-21.

Derrida, Jacques. L'écriture et la différance. Paris: Éditions du Seuil, 1967: 440.

De la gramatología. Buenos Aires: Siglo Veintiuno Editores, 1971: 397.

La reconstrucción en las fronteras de la filosofía. Traducción: Patricio Peñalver. Barcelona: Paidós, 1979: 128.

Einstein, Albert. La relatividad. Traducción: Ute Schmidt de Cepeda. México: Grijalbo, 1991: 204.

Fokkema, D. W. e Ibsch, Elrud. Teorías de la literatura del siglo XX. Cuarta edición. Traducción: Gustavo Domínguez. Madrid: Ediciones Cátedra, 1992: 242.

Garaudy, Roger. El pensamiento de Hegel. Traducción: Francisco Monge. Barcelona: Seix Barral, 1974: 312.
Gorski, D. P. y Tavants, P. V. Lógica. Décimo quinta edición. Traducción: Augusto Vidal. México: Grijalbo, 1970: 320.

Gortari, Eli de. Ejercicios y problemas de lógica. México: Ediciones Océano, 1984: 348.

Hawking, Stephen W. Historia del tiempo. Del Big Bang a los agujeros negros. $3^{\mathrm{a}}$ edición. Traducción: Miguel Ortuño. México: Editorial Crítica, 1988: 248.

Hegel, G. W. Lógica. 2a edición. Traducción: Antonio Zozaya. Madrid: Editorial Ricardo Aguilera, 1973: 374.

- Ciencia de la lógica. $4^{\mathrm{a}}$ edición. Traducción: Augusta y Rodolfo Mondolfo. Buenos Aires: Ediciones Solar y Librería Hachette, 1976: 756.

Kant. Crítica de la razón pura (tomo II). $7^{\mathrm{a}}$ edición. Traducción: José del Perojo. Buenos Aires: Editorial Losada, 1973: 368.

Kojève, Alexandre. La dialéctica de lo real y la idea de muerte en Hegel. Traducción: Juan José Sebreli. Buenos Aires: Editorial La Pléyade, 1972: 192.

Kopnin, P. V. Lógica dialéctica. Traducción: Lidya Kuper. México: Grijalbo, 1966: 564.

Lefebvre, Henri. El materialismo dialéctico. Traducción: Rubén A. N. Laporte. Buenos Aires: Editorial La Pléyade, 1974: 192.

Lógica formal, lógica dialéctica. $4^{\mathrm{a}}$ edición. Traducción: María Esther Benitez. México: Siglo Veintiuno Editores, 1976: 350.

Lukács, György. Sociología de la literatura. $2^{a}$ edición. Traducción: Michael FaberKaiser. Barcelona: Ediciones Península, 1968: 510. 
- Historia y conciencia de clase. Traducción: Manuel Sacristán. México: Grijalbo, 1975: 358.

Macho Stadler, Marta. “Qué es la topología?” Sigma 20, febrero, 2002: 63-77.

Mora Burgos, Gerardo. "Posibilidad y necesidad de una hermenéutica materialista”. Revista de Filosofía 29. 69, junio. Universidad de Costa Rica. 1991: 15-22.

NagyZekmi, Silvia. "Estrategias poscoloniales: la deconstrucción del discurso eurocéntrico". Cuadernos Americanos 1. 97,enero-febrero. Año 27. 2003: 1120.

Quesada Soto, Álvaro. "La narrativa costarricense del último tercio de siglo". Letras 32. 2000: 17-43.
Roth, Wolff-Michael. "A dialectical materialist reading of the sign". Semiotica. 2006: $160-1 / 4 ; 141-171$.

Seoane, Javier. "La Escuela de Frankfurt y los Posmodernos: un diálogo posible". Revista Venezolana de Economía y Ciencias Sociales 6.2, mayo-agosto, 2000: 19321. En: <http://www.revele.com.ve/pdf/ revista_venezolana_de_economía_y_ ciencias_sociales/vol10_n1/pag39.pdf> [Recuperado el 1 de abril del 2005]

Villalobos Alpizar, Iván. "Algunas consideraciones en torno al tema de la intencionalidad y el yo en Husserl". Revista de Filosofía 37. 93,diciembre. Universidad de Costa Rica. 1999: 405-412.

Villegas, Juan. La interpretación de la obra dramática. Santiago de Chile: Editorial Universitaria, 1971. 